\title{
RIG-I suppresses the migration and invasion of hepatocellular carcinoma cells by regulating MMP9
}

\author{
ZHIKUI LIU*, CHANGWEI DOU*, YULI JIA, QING LI, XIN ZHENG, \\ YINGMIN YAO, QINGGUANG LIU and TAO SONG \\ Department of Hepatobiliary Surgery, First Affiliated Hospital of Medical College \\ of Xi'an Jiaotong University, Xi'an, Shaanxi 710061, P.R. China
}

Received November 7, 2014; Accepted December 22, 2014

DOI: $10.3892 /$ ijo.2015.2853

\begin{abstract}
The retinoic acid-induced protein I (Rig-I/Ddx58), (RIG-I) initiates a signaling cascade that induces innate immune defences which is associated with the production of type I interferons (IFNs) and inflammatory cytokines to establish an antiviral state. Aberrant RIG-I signaling leads to inflammation, autoimmune diseases and cancer. However, the role of RIG-I in hepatocellular carcinoma (HCC) is still unknown. Here, we observed that RIG-I expression was downregulated in HCC tissues and loss of RIG-I expression was correlated with poor clinicopathological features. Additionally, we demonstrated that patients with positive RIG-I expression had a better 3-year survival and RIG-I was an independent factor for predicting the prognosis of HCC patients. Elevated RIG-I expression inhibited the proliferation, migration, and invasion of HCC. Inhibiting RIG-I with its specific siRNA was able to attenuate the malignant behavior of HCC cells. Moreover, RIG-I inhibited the invasive behavior through downregulating matrix metalloproteinase-9 (MMP9). Mechanistically, RIG-I enhances IFN- $\alpha$ response by amplifying IFN- $\alpha$ effecter signaling via strengthening STAT1 activation. Addressing this pathway, we identified that RIG-I may serve as a prognostic marker and that MMP9 may be a potential target of RIG-I in HCC.
\end{abstract}

\section{Introduction}

Hepatocellular carcinoma (HCC) is the sixth most common malignancy worldwide and is among the leading common causes of global cancer related mortality, especially in China (1). Despite considerable diagnostic and therapeutic

Correspondence to: Dr Qingguang Liu and Dr Tao Song, Department of Hepatobiliary Surgery, The First Affiliated Hospital of Xi'an Jiaotong University, 277 Yanta West Road, Xi'an, Shaanxi 710061, P.R. China

E-mail: liuqingguang@vip.sina.com

E-mail: 13572431619@163.com

"Contributed equally

Key words: retinoic acid-induced protein I, hepatocellular carcinoma, interferon- $\alpha$ therapeutic response, matrix metalloproteinase- 9 advances in recent years, the overall prognosis of HCC patients remains unsatisfactory because of the high rate of recurrence and metastasis. Patients who have undergone radical resection have a high rate of relapse, even when treated with adjuvant systemic and targeted therapies, including transcatheter arterial chemoembolization and sorafenib. Substantial functional studies of pro-oncogenes and tumor suppressor genes have greatly contributed to the advance of knowledge on the molecular mechanism of HCC development (2). However, the validation of biomarkers, which may help to predict regional and distant invasion and metastasis, is still an unresolved problem. Thus, it is critical to clarify the molecular mechanisms underlying the invasive and metastatic potential of HCC and identify a molecular biomarker that can predict the prognosis of patients.

The retinoic acid-induced protein I (Rig-I, encoded by $\mathrm{Ddx} 58$ ) contains two $\mathrm{N}$-terminal caspase recruitment domains (CARDs), a central DExH box helicase/ATPase and a C-terminal regulatory domain (CTD). The CTD can recognize cytoplasmic viral RNA such as vesicular stomatitis virus (VSV) and Sendai virus (3). Through activating downstream pathways, CARDs serve as a critical sensor for host recognition of RNA virus to activate innate immune cells such as dendritic cells. CARDs also directly induce expression of type I interferon (IFN) and apoptosis in tumor cells (4). Thus, here we focus on whether RIG-I plays a role in the invasion and metastasis of HCC. We also investigated whether the signaling pathway RIG-I-IFN functioned in HCC and discovered a specific downstream molecule of this pathway, which was proved to be an effective approach to controlling HCC.

For the adjuvant therapy, interferon- $\alpha$ (IFN- $\alpha$ ) can prolong HCC patients survival $(5,6)$ and has been approved by the FDA as a therapeutic method to treat $\mathrm{HCC}$, either in single therapy or combination therapy with other anticancer drugs $(5,6)$. It has various biological properties, including immunomodulation, antiviral effect and antiproliferative activity (7). Considerable research has been undertaken to clarity the molecular mechanisms responsible for its versatile biological functions (8). In the classical signaling pathway, IFN- $\alpha$ binds to the cell-surface receptors and subsequently activates the JAK (Janus activated kinase)-STAT (signal transducer and activator of transcription) signaling cascade (9). Phosphorylated Stat dimers finally translocate into the nucleus where they activate target genes 
which are involved in multiple cellular processes (10), including cell proliferation, apoptosis (11), carcinogenesis (12), and immune inhibition in tumor microenvironment (13). As a consequence of these different functions, it was revealed that the coordination and cooperation of multiple distinct signaling cascades are required for response to interferon. Thereby, these induce transcription of interferon-stimulated genes (ISGs) that are involved in anti-proliferation, anti-invasion and antimetastasis activity $(14,15)$. MMP9 is one of the ISGs and is involved in proteolytic degradation of the extracellular matrix, which is a critical step in tumor invasion. Aberrant expression of MMP9 is strongly correlated with malignant tumor grade $(16,17)$. Moreover, RIG-I can enhance response to IFN- $\alpha$ therapy in HCC. The induced RIG-I expression on IFN- $\alpha$ stimulation may be a positive feedback of IFN- $\alpha$ response to amplify IFN- $\alpha$ effector signaling and ensure a longtime IFN- $\alpha$ efficacy (18). However, the detailed role that RIG-I plays in IFN- $\alpha$ signaling pathway is still unknown.

In this study, we investigated whether RIG-I could regulate MMP9 expression in HCC cells. In addition, we investigated the roles of RIG-I in modulating the efficacy of IFN- $\alpha$ and explored its possibility as prognostic biomarker and its potential applications as a predictive element for therapeutic interventions. Our results indicated that RIG-I suppressed MMP9 expression through IFN- $\alpha$-JAK-STAT1 pathway, and RIG-I strengthened the IFN-JAK-STAT effector signaling by promoting STAT1 activation and then enhanced therapeutic response to IFN- $\alpha$ therapy in HCC. Therefore, the RIG-I and IFN- $\alpha$ expression may be feedback to ensure longtime IFN- $\alpha$ efficacy and RIG-I expression may have tumor-suppressive effects in cancer cells.

\section{Materials and methods}

Ethics statement. Written informed constent was obtained from all patients recruited in this study. The ethics committee of our hospital approved all protocols according to the Helsinki Declaration of 1975. In addition, all experimental protocols were approved by the institutional animal care and use committee of our hospital.

Patients and specimens. Cancerous tissues and adjacent noncancerous ( $>2 \mathrm{~cm}$ distance to the resection margin) hepatic parenchyma were obtained from $80 \mathrm{HCC}$ patients who underwent curative resection surgery from 2010 to 2011 in the First Affiliated Hospital of Xi'an Jiaotong University, Department of Hepatobiliary Surgery. The patients did not have any other malignancies and did not receive chemotherapy or radiotherapy before operation. Fresh tissues were frozen at $-80^{\circ} \mathrm{C}$ for RNA and protein analyses. One section of each specimen was stained with hematoxylin and eosin (H\&E) staining for pathological diagnosis. Samples were obtained from 63 men and 17 women aged 36-78 years with a median age of 55 years. Clinical data were collected from the medical records. Informed consent was obtained from all patients before the study began. The Investigation and Ethics Committee of our hospital approved all protocols.

Immunohistochemical staining. Tissues were fixed in formalin and embedded in paraffin before sectioning. Briefly, the sections $(4 \mu \mathrm{m})$ were deparaffinized in xylene and rehydrated by stepwise washes in decreasing ethanol, quenched for endogenous peroxidase in 3\% hydrogen peroxide, followed by boiling in retrieval solution to expose antigens and then slides were incubated with the primary antibody against RIG-I (Cell Signaling Technology) overnight at $4^{\circ} \mathrm{C}$. The reaction was visualized with diaminobenzidine and counterstained with hematoxylin, which resulted in a brown-colored stain at the site. All slides were stained with hematoxylin and eosin for histologic analysis and observed by two pathologists independently to evaluate the results. The measurement of positive staining density was performed by an immunohistochemical score and the percentage of tumor cells showing specific staining. The intensity was assessed by four grades: 0 for none; 1 for weak; 2 for moderate; 3 for strong. The percentage of positive cells was divided into four degrees: 0 for $<5 \%$; 1 for $5-25 \%$; 2 for $26-50 \%$; 3 for $>50 \%$. The total scores $>1$ were defined as positive. The overall intensity and percentage were evaluated at 10 independent fields (x400).

Cell culture. The human HCC cell lines Huh7, HepG2, Hep3B, SMMC-7721, Bel-7402 and normal human liver permanent cell line LO2 were obtained from Shanghai Institute of Cell Biology, Chinese Academy of Sciences, Shanghai, China. All of them were maintained in high-glucose DMEM supplemented with $10 \%$ heat-inactivated fetal bovine serum (FBS). DMEM medium, FBS and trypsin/EDTA were purchased from Invitrogen Co. (Carlsbad, CA, USA). All cell lines were cultured at $37^{\circ} \mathrm{C}$ in a humidified incubator in $5 \% \mathrm{CO}_{2}$.

Quantitative reverse transcription-polymerase chain reaction $(q R T-P C R)$. Total RNA was extracted from HCC cell lines and tissues with TRIzol reagent (Invitrogen) according to the manufacturer's protocol, and reversed transcribed with RevertAid ${ }^{\mathrm{TM}}$ first-strand cDNA synthesis kit (Fermentas Burlington, ON, Canada). RIG-I mRNA levels were determined by qPCR using SYBR Premix Ex Taq (Takara, Dalian, China) and normalized with GAPDH using the following primers: RIG-I forward, 5'-TGT GCT CCT ACA GGT TGT GGA-3', and reverse, 5'-CAC TGG GAT CTG ATT CGC AAA A-3'; GAPDH forward, 5'-CAA GGT CAT CCA TGA CAA CTT TG-3', and reverse, 5'-GTC CAC CAC CCT GTT GCT GTA G-3'. The primers were synthesized by GenePharma Co., Ltd. (Shanghai, China). The PCR products were visualized on $2 \%$ agarose gel and quantitative RT-PCR was performed at least three times per sample. The relative gene expression was calculated with the 2- $\Delta \mathrm{Ct}$ method. Three independent experimental replicates were performed.

Western immunoblotting. Cells and tissues were lysed on ice in RIPA lysis buffer according to manufacturer-provided instructions. The protein concentration was quantified with the BCA reagent (Hercules, CA, USA). Equal amounts of protein were divided for electrophoresis on an 8-15\% SDS-PAGE gel and then transferred to a nitrocellulose membrane (Millipore, Billerica, MA, USA). After blocking with 5\% non-fat milk for $1 \mathrm{~h}$, the membranes were incubated with antibodies, respectively, overnight at $4^{\circ} \mathrm{C}$, after washed 3 times with TBS $/ 0.1 \%$ Tween-20, blots were incubated with horseradish peroxidaseconjugated secondary antibodies (Bio-Rad, Hercules, CA, 
USA). Chemiluminescence detection was performed according to the manufacturer's instructions (Bio-Rad). $\beta$-actin was measured to control for equal loading.

Cell transfection and clone selection. The pGFP-RIG-I plasmid was constructed by Shanghai GeneChem Co. Transfection was performed using Lipofectamine ${ }^{\mathrm{TM}} 2000$ (Invitrogen) according to the manufacturer's instructions. At $48 \mathrm{~h}$ after transfection, the cells were collected for further assessment. Stable transfection clones were obtained after 3-week selection with Geneticin (G418) from Invitrogen at a dose of $600 \mu \mathrm{g} / \mathrm{ml}$. Stably transfected clones were determined by qRT-PCR and immunoblotting for the level of RIG-I expression.

RNA interference. The human RIG-I specific siRNA were 5'-GGU GGA GGA UAU UGC AAC UTT-3' (sense) and 5'-AGU UGC AAU AUC CUC CAC CTT-3' (antisense); the scrambled control RNA sequences were 5'-UUC UCC GAA CGU GUC ACG UTT-3' (sense) and 5'-ACG UGA CAC GUU CGG AGA ATT-3' (antisense). siRNA was synthesized from Genechem (Shanghai, China). siRNA duplexes were transfected at a final concentration of $10 \mathrm{nM}$.

Colony formation assay. Five hundred LO2, Huh7, HepG2, Hep3B, SMMC-7721, Bel-7402 cells were placed in 6-well plates and maintained in DMEM containing 10\% FBS. When the single cell grows to a 50-cell colony, colonies were fixed with $20 \%$ methanol and stained with $0.1 \%$ crystal violet at room temperature for $15 \mathrm{~min}$.

Wound healing assay. Briefly, the HCC cells were grown and transfected with empty vector and pGFP-RIG-I plasmid for $48 \mathrm{~h}$. The cells were collected and seeded into 6-well plates and cultured to $>90 \%$ confluency in a monolayer. Scratch wound were made using a $200-\mu 1$ pipette tip. Images of wounds were taken with a phase contrast microscope at 0,24 and $48 \mathrm{~h}$. The distance from one side to the other was measured using Image Pro-Plus 6.0 software (Media Cybernetics, USA). For each experiment, 5 visual fields and 2 repeated wells were measured with three replications.

MTT assay. Proliferation was determined using the MTT assay (Roche Diagnostics, USA). After 48-h incubation following pEGFP-RIG-I plasmid (Gendicine, Sibiono GeneTech Co., Ltd., Shenzhen, China) addition, cells were cultured further for $0-72 \mathrm{~h}$, as before, the effect of IFN- $\alpha$ (Peprotech) on HCC cell growth was determined. The absorbance of the samples was measured using a model 550 microplate reader (Bio-Rad Laboratories), at a wavelength of $570 \mathrm{~nm}$ corrected to $655 \mathrm{~nm}$. Three experimental replicates were performed.

Invasion assay. Cell invasion assays were performed using 24-well transwells (8- $\mu \mathrm{m}$ pore size; Millipore) coated with Matrigel (BD Biosciences, San Jose, CA, USA). In total, $1 \times 10^{5}$ cells were suspended in $100 \mu 1$ DMEM and added to the upper chamber, and $600 \mu$ l DMEM containing 20\% FBS was placed in the lower chamber. After $24 \mathrm{~h}$ of incubation, the cells remaining in the top surface of Matrigel membrane was swabbed carefully and the membrane was fixed with $4 \%$ paraformaldehyde for $10 \mathrm{~min}$ and stained with $0.1 \%$ crystal violet solution for $10 \mathrm{~min}$. Cells in five microscopic fields were counted and photographed randomly to obtain the mean cell number in each membrane. All experiments were performed in triplicate.

Immunofluorescence staining. After designated treatment, HCC cells were fixed with $4 \%$ paraformaldehyde for $10 \mathrm{~min}$ at room temperature, permeabilized in $0.5 \%$ Triton X-100 for $10 \mathrm{~min}$, and blocked in $1 \%$ BSA for $1 \mathrm{~h}$. Fixed cells were then incubated with the RIG-I (1:100) primary antibody at $4^{\circ} \mathrm{C}$ overnight. Cells were washed and incubated with goat antirabbit FITC (green) IgG antibody (ZSGB-BIO Inc., Beijing, China) at 1:100 dilution for $60 \mathrm{~min}$. Nuclei were stained with DAPI for $5 \mathrm{~min}$. The cells were visualized by a fluorescent microscope (Nikon, Japan) using appropriate excitation and emission spectra at x400 magnification.

In vivo experiments. Ten BALB/ca female nude mice (Shanghai Institute of Materia Medica, Chinese Academy of Science), 4-6-week-old, were kept in laminar-flow cabinets under specific pathogen-free conditions, and handled according to the recommendations of the National Institutes of Health guidelines for care and use of laboratory animals. For the tumor challenge, $2 \times 10^{6}$ Hep3B-pEGFP-RIG-I plasmid or Hep3B-mock tumor cells in $100 \mu \mathrm{l}$ of Matrigel were injected subcutaneously into the flanks of nude mice, and tumor growth was monitored each week. There were five animals in each group. The mice were sacrificed on day 28 after implantation. Tumor tissues were prepared for western blot analysis and immunostaining.

Statistical analysis. Statistical analysis was performed with SPSS software (version 16.0, SPSS, Chicago, IL, USA). All data are shown as the mean \pm SD. Differences between two groups were estimated with Student's t-test and ANOVA. Statistical significance was defined as $\mathrm{P}<0.05$.

\section{Results}

RIG-I expression is significantly decreased in HCC. We examined $80 \mathrm{HCC}$ samples and corresponding adjacent noncancerous hepatic tissues for RIG-I mRNA expression using semi-quantitative RT-PCR. The average RIG-I/GAPDH level in HCCs was significantly lower than that in non-cancer tissues (Fig. 1A). Consistent with its RNA expression, RIG-I protein expression was also obviously lower in HCC tissues (Fig. 1B). Immunohistochemical study showed that RIG-I protein was detectable in both cancer and non-cancer tissues (Fig. 1C). However, the positive immunoreactivity was correlated with $\sim 85 \%$ non-cancerous tissues (68/80), while only $40 \%$ in HCC cases (32/80) showed positive protein staining. The RIG-I protein staining was observed in the cytoplasm, and tended to be greater in the non-cancer cells after analysis of the results of IHC scores using Mann-Whitney test $\left(\chi^{2}=34.56, \mathrm{P}<0.01\right)$. We concluded that RIG-I expression was significantly decreased in $\mathrm{HCC}$.

RIG-I expression is a predictor of HCC progression and prognosis of HCC patients. To determine the potential role of RIG-I in HCC progression, we evaluated the correlation between RIG-I expression and histopathological parameters in patients 

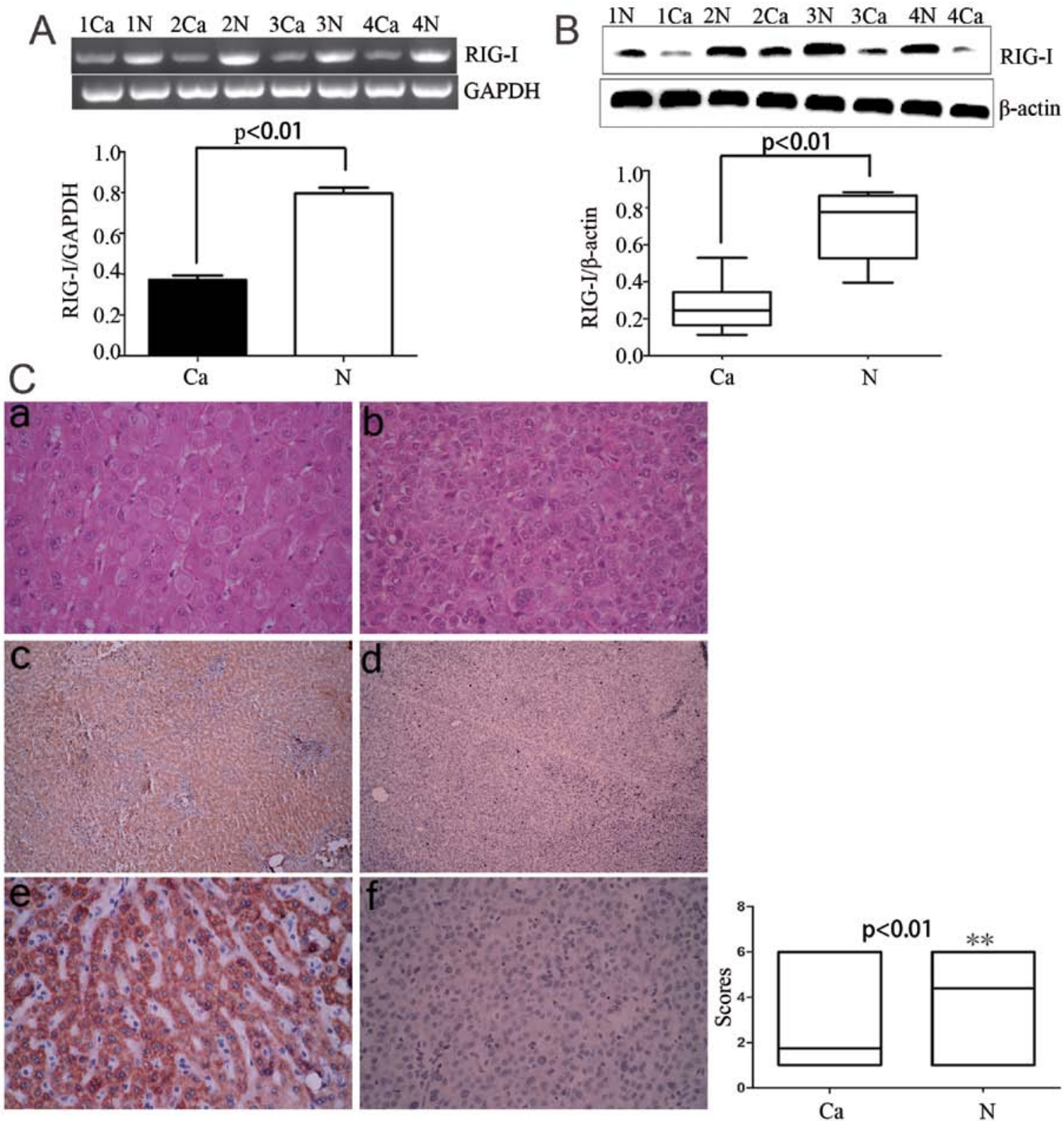

Figure 1. RIG-I expression is significantly decreased in HCC, RT-PCR analysis, western blotting, immunohistochemical staining of representative tissues from 80 HCC samples. (A) Ratio of average RIG-I/GAPDH expression in HCC compared with corresponding non-cancer hepatic tissues. (B) The average RIG-I/ $\beta$ actin level in HCCs. (C) Immunohistochemistry using anti-RIG-I antibodies; representative IHC staining of RIG-I in adjacent normal tissues (c and e) and in HCC tissues (d and f). (a and b) For pathological diagnosis in H\&E; (c and d) original magnification x100; (c) high RIG-I expression; (d) low RIG-I expression; (e and f) x400 magnification; (e) high RIG-I expression; (f) low RIG-I expression.
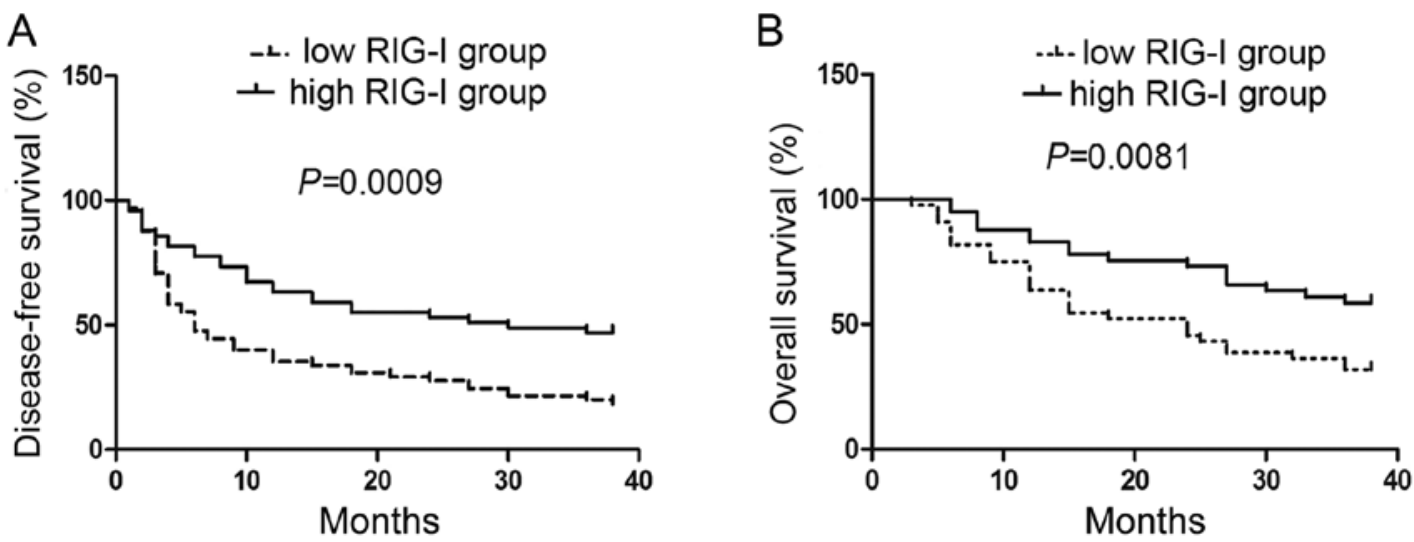

Figure 2. Survival rates of HCC patients. (A) Disease-free survival rates of patients with HCCs. (B) Overall survival rates of patients with HCCs. High expression of RIG-I was significantly associated with improved prognosis. 
Table I. Correlations between RIG-I expression and clinicopathological characteristics.

\begin{tabular}{lcccc}
\hline & & \multicolumn{2}{c}{ Expression level } & \\
\cline { 3 - 4 } Clinical & Cases & Positive & Negative & P-value \\
parameters & $(\mathrm{n})$ & $(\mathrm{n}=32)$ & $(\mathrm{n}=48)$ & \\
\hline
\end{tabular}

Age (years)

$\begin{array}{lllll}<65 & 46 & 17 & 29 & 0.645 \\ \geq 65 & 34 & 15 & 19 & \end{array}$

Gender

Male

Female

63

$17-10$

41

0.097

Tumor size $(\mathrm{cm})$

\begin{tabular}{|c|c|c|c|c|}
\hline$<5$ & 41 & 22 & 19 & \\
\hline$\geq 5$ & 39 & 10 & 29 & \\
\hline Tumor number & & & & 0.783 \\
\hline Solitary & 63 & 26 & 37 & \\
\hline Multiple & 17 & 6 & 11 & \\
\hline \multicolumn{5}{|l|}{ Edmondson } \\
\hline $\mathrm{I}+\mathrm{II}$ & 39 & 19 & 15 & $0.020^{\mathrm{a}}$ \\
\hline III+IV & 41 & 13 & 33 & \\
\hline TNM stage & & & & $0.003^{*}$ \\
\hline I-II & 36 & 21 & 15 & \\
\hline III-IV & 44 & 11 & 33 & \\
\hline Capsular infiltration & & & & 0.402 \\
\hline Present & 64 & 24 & 40 & \\
\hline Absent & 16 & 8 & 8 & \\
\hline Vascular invasion & & & & $0.007^{\mathrm{a}}$ \\
\hline Present & 61 & 19 & 42 & \\
\hline Absent & 19 & 13 & 6 & \\
\hline $\operatorname{AFP}(\mathrm{ng} / \mathrm{ml})$ & & & & 0.633 \\
\hline$<400$ & 53 & 20 & 33 & \\
\hline$\geq 400$ & 27 & 12 & 15 & \\
\hline HBsAg & & & & 0.773 \\
\hline Positive & 71 & 28 & 43 & \\
\hline Negative & 9 & 4 & 5 & \\
\hline
\end{tabular}

${ }^{\mathrm{a}} \mathrm{P}<0.05$. with HCC. Clinical association analysis using a Pearson $\chi^{2}$ test revealed that the reduced RIG-I expression in HCC tissues was correlated with large tumor size ( $\geq 5 \mathrm{~cm}$; $P=0.013$ ), venous infiltration $(\mathrm{P}=0.007)$, high histological grade (EdmondsonSteiner grade III + IV; $\mathrm{P}=0.020)$ and advanced tumor stage (TNM stage III + IV; P=0.003) (Table I). Its expression level was not correlated with gender, age, HBV infection or histological differentiation degree. These results indicate a potential role of RIG-I expression in inhibiting aggressive phenotypes in HCC.

Next, as RIG-I expression is significantly decreased in HCC, we further assessed whether the level of RIG-I in tumor tissues predicted the prognosis of patients. We followed up the HCC patients recruited in this study to obtain prognostic information of $73 \mathrm{HCC}$ cases $(91.3 \%)$. The median duration of follow-up was 26 months (range, 9-38 months). Using the median IHC score of RIG-I in HCC tissues as the cut-off value, after analyzing them with the Kaplan-Meier survival curve, we found that patients with high RIG-I expression had improved disease-free survival and overall survival (Fig. 2, $\mathrm{P}<0.01$ ). Multivariate analysis that enrolled all of the significant clinical factors for OS and DFS indicated that RIG-I positive expression (Table II) was an independent prognostic factor for HCC patients. These results suggested that RIG-I expression was effective in predicting prognosis of patients.

RIG-I expression in HCC cell lines. We performed RT-PCR and qRT-PCR to analyze the differential mRNA levels of RIG-I among HCC cell lines including SMMC-7721, Bel-7402, Hep3B, Huh7 and HepG2, and the normal liver LO2 cells. We found that compared with $\mathrm{LO} 2$ cell line, all five HCC cell lines expressed significantly lower levels of RIG-I mRNA [Fig. 3A (RT-PCR) and B (qRT-PCR), P<0.01]. Among these HCC cell lines, RIG-I was noted to be highest in SMMC-7721 and lowest in Hep3B. Result of western blotting was consistent with that of RT-PCR (Fig. 3C and D, P<0.01). We then visualized the cellular location of RIG-I in these 6 HCC cell lines using immunofluorescence (Fig. 3E). Consistent with the immunohistochemical data from the HCC tissues, the single cell-labeling revealed that positive staining for RIG-I protein was intense in the cytoplasm of all the tested HCC cell lines.

RIG-I inhibits proliferation, migration and invasion of HCC cells. To investigate the effect of RIG-I on HCC cells, we measured RIG-I mRNA expression level and assessed

Table II. Multivariate Cox regression analysis of 3-year disease-free and overall survival of hepatocellular carcinoma.

\begin{tabular}{|c|c|c|c|c|c|c|}
\hline \multirow[b]{2}{*}{ Variables } & \multicolumn{3}{|c|}{ Overall survival } & \multicolumn{3}{|c|}{ Disease-free survival } \\
\hline & HR & $95 \% \mathrm{CI}$ & P-value & HR & $95 \% \mathrm{CI}$ & P-value \\
\hline RIG-I & 3.612 & $1.109-7.987$ & $0.003^{\mathrm{a}}$ & 2.358 & $1.082-4.976$ & $0.009^{\mathrm{a}}$ \\
\hline Vascular nvasion & 2.741 & $1.312-5.736$ & $0.007^{\mathrm{a}}$ & 2.592 & $1.273-5.286$ & $0.009^{\mathrm{a}}$ \\
\hline Edmondson grade & 3.423 & $1.348-9.129$ & $0.014^{\mathrm{a}}$ & 2.217 & $1.081-4.629$ & $0.048^{\mathrm{a}}$ \\
\hline TNM stage & 4.201 & $1.527-10.619$ & $0.027^{\mathrm{a}}$ & 7.314 & $2.018-23.69$ & $0.030^{\mathrm{a}}$ \\
\hline
\end{tabular}

HR, hazard ratio; CI, confidence interval. a'Statistically significant. 

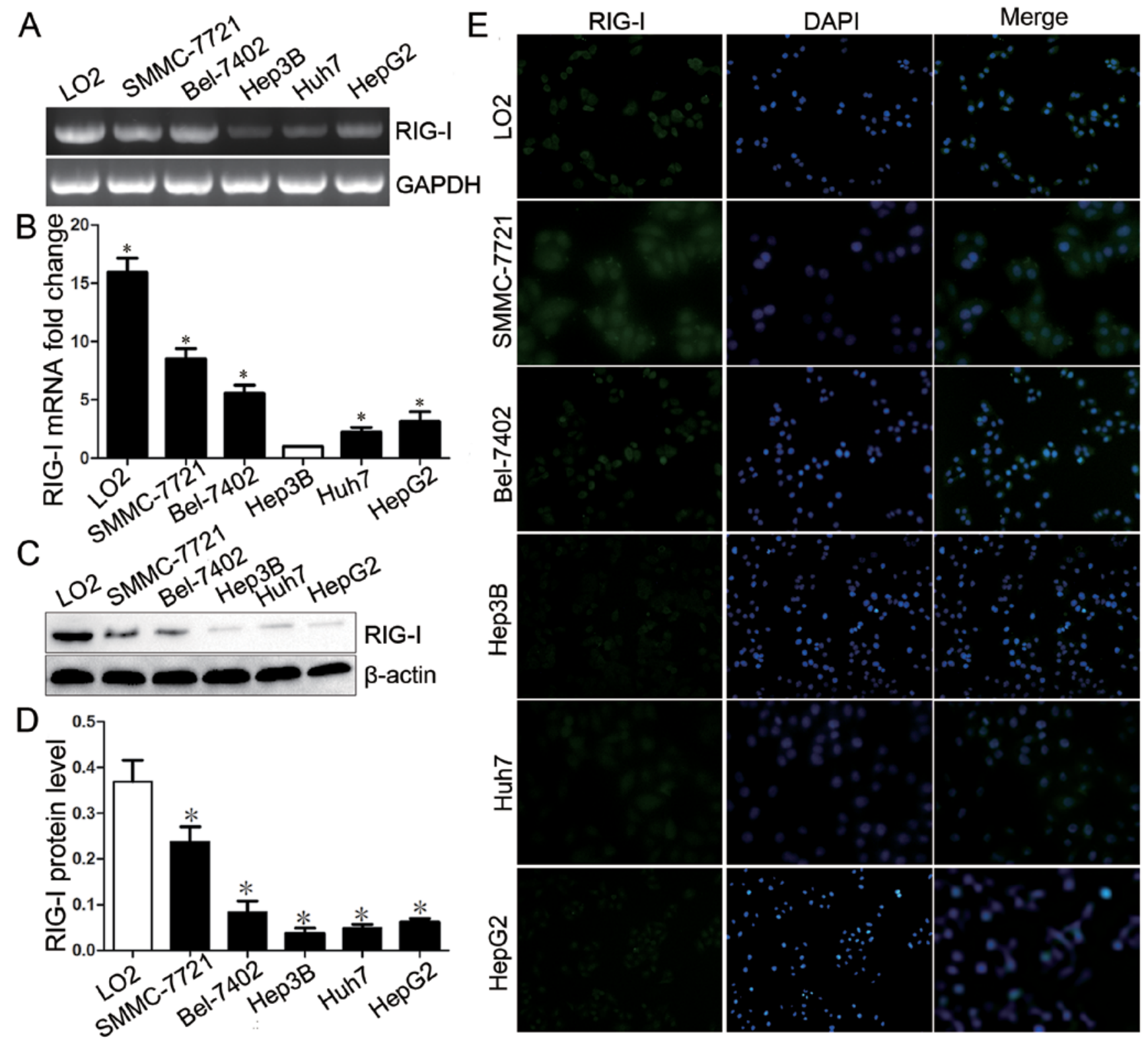

Figure 3. Expression of RIG-I mRNA and protein in HCC cell lines. The cells of five hepatoma cell lines and a normal liver cell line were subjected to (A) RT-PCR, (B) qRT-PCR, (C) western blotting and (D) summarized as RIG-I protein expression compared to $\beta$-actin. (E) The location of RIG-I proteins within cells of the six cell lines determined by single-labeled immunofluorescence assay. Green color indicates RIG-I-positive, blue color indicates the nuclear staining. ${ }^{*} \mathrm{P}<0.01$.

colony formation ability of LO2 cells and the HCC cell lines including SMMC-7721, Bel-7402, Hep3B, Huh7 and HepG2. LO2 expressing the highest RIG-I mRNA levels showed the poorest ability to form colonies (Figs. 3B and 4A). In addition, RIG-I mRNA expression level was negatively correlated with the number of cell colonies $(\mathrm{t}=-4.608, \mathrm{r}=-0.893, \mathrm{P}=0.016$, Fig. 4B).

Next, we enforced RIG-I expression in Hep3B and Huh7 cells which expressed RIG-I at low level employing RIG-I expressing plasmid which contained GFP. Immunofluorescence confirmed the successful transfection of RIG-I expressing plasmid into these cells (Fig. 4C). qRT-PCR and western blotting results confirmed that RIG-I mRNA and protein level were significantly upregulated in these two cell lines after transfection (Fig. 4D and E). Then, we evaluated the functional role of RIG-I on tumor cells. After stably transfected cells were individually selected, since the presence of RIG-I reduces the ability of cell colony formation, we investigated whether the overexpression of RIG-I mRNA affected cell proliferation. MTT (3-(4,5-dimethylthiazol-2-yl)-2,5-diphenyl tetrazolium bromide) assay revealed that elevated RIG-I significantly negatively affected the viability of Hep3B and Huh7 cell proliferation (Fig. 4F). Then we explored whether RIG-I regulated HCC cell migration and invasion. First, we examined the role of RIG-I in tumor cell migration using wound scratch assay. As illustrated in Fig. 4G, enforced expression of RIG-I significantly inhibit wound closure in Hep3B and Huh7 cells. To further validate these observations, we examined the effects of RIG-I on HCC cell migration using the transwell chamber without Martrigel. Indeed, tumor cells derived from RIG-I-transfectants displayed less ability of migration, compared with those derived from control vector 

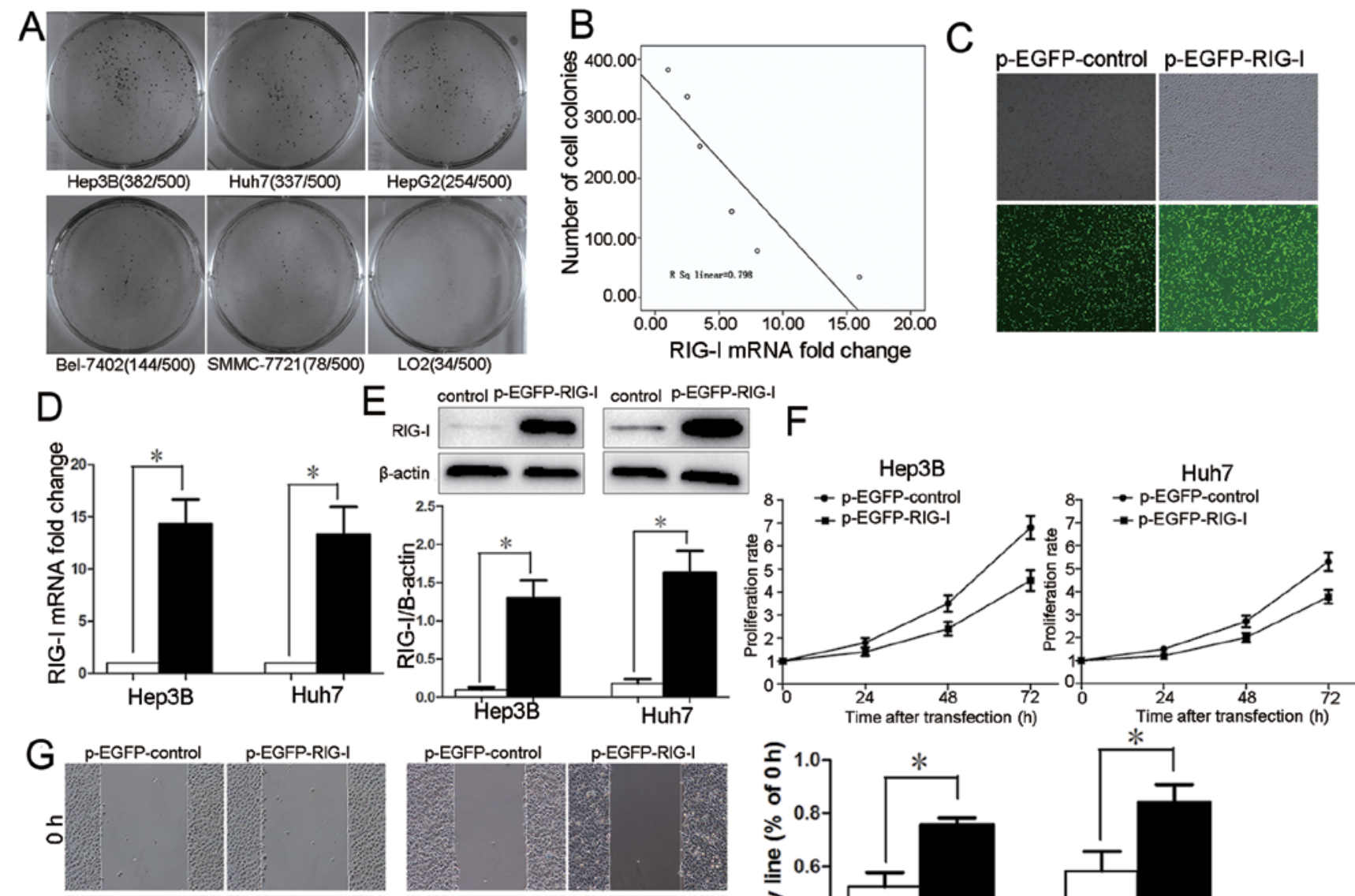

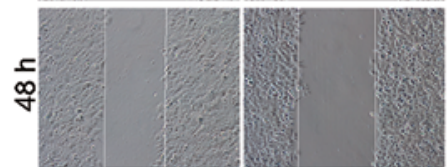

Нер3В

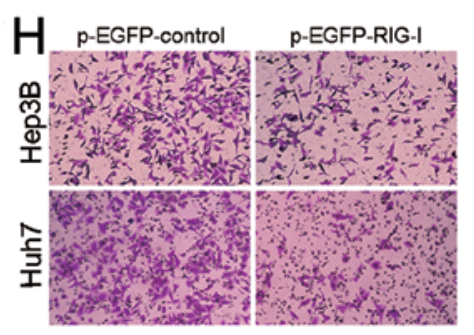

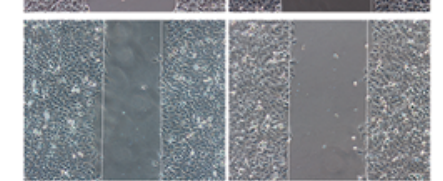

Huh7

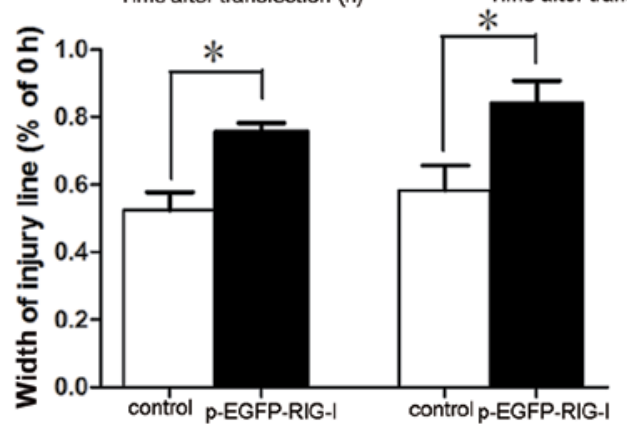

Huh7 Hep3B
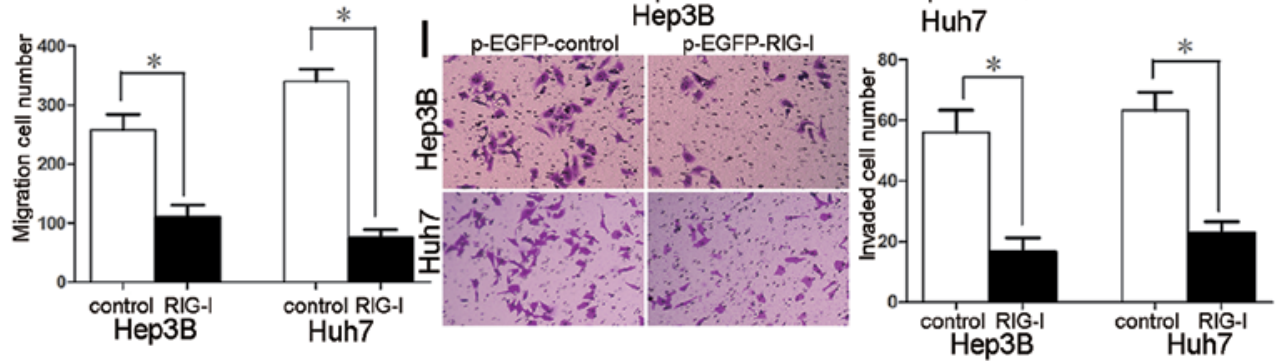

Figure 4. RIG-I inhibits proliferation, migration and invasion in HCC cells. (A) Colony formation assay quantification with HCC cell lines. (B) Correlation plot for RIG-I mRNA levels and colony number. Values are depicted as mean \pm standard error. (C) Transfection efficiency of two groups (control plasmid, and RIG-I plasmid) of Hep3B cells. The optical micrographs are in the upper line and the fluorescence micrographs are in the lower line. (D and E) qRT-PCR, western blotting confirmed overexpression in Hep3B and Huh7 cells treated with RIG-I plasmid. (F) MTT assay showed that upregulation RIG-I significantly influence the proliferation rates in Hep3B and Huh7 cells. (G) Wound healing assay indicated that RIG-I upregulation inhibited cell migration. (H and I) Elevated RIG-I expression attenuated the migration and invasion in Hep3B and Huh7 cells. Cells transfected with p-EGFP-control and p-EGFP-RIG-I cells were seeded into the Transwell chamber coated without or with Matrigel and incubated in DMEM containing 10\% FBS for 24 h. Representative images and the number of migrated or invaded cells are shown. Results are presented as mean \pm SEM $(n=3)$. . $P<0.01$.

(Fig. 4H). Next, we determined the role of RIG-I in tumor cell invasion. We performed these experiments using transwell chamber with Matrigel. As illustrated in Fig. 4I, upregulation of RIG-I expression significantly decreased the number of cells that invaded through Matrigel in comparison with that derived from the vector.

To further identify these observations, we confirmed the results in an opposite method by transfecting specific siRNA to knock down RIG-I in SMMC-7721 and Bel-7402 cells which expressed high levels of RIG-I endogenously. qRT-PCR and western blotting results confirmed that RIG-I mRNA and protein level were significantly downregulated by RIG-I siRNA in these two cell lines (Fig. 5A and B). Next, we investigated whether RIG-I deregulation influenced tumor cell proliferation. Therefore, proliferation was assessed in SMMC-7721 and Bel7402 cells. In these cells, obviously increased proliferation rates were observed (Fig. 5C). At the same time, downregulation of RIG-I remarkably promoted the wound 
A
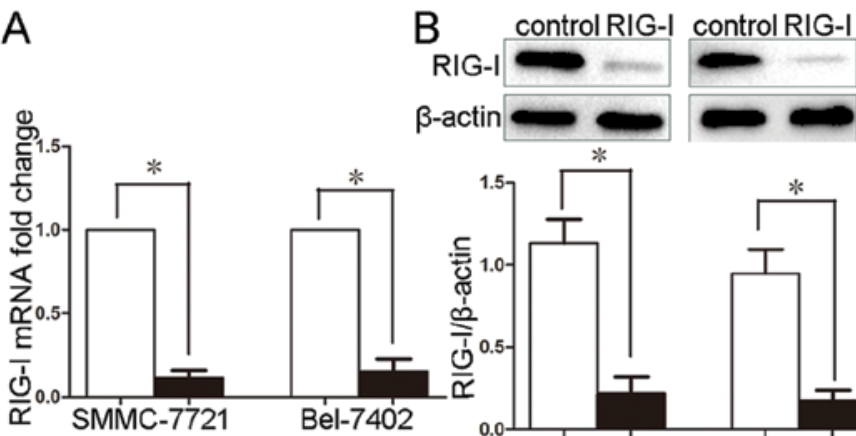

C
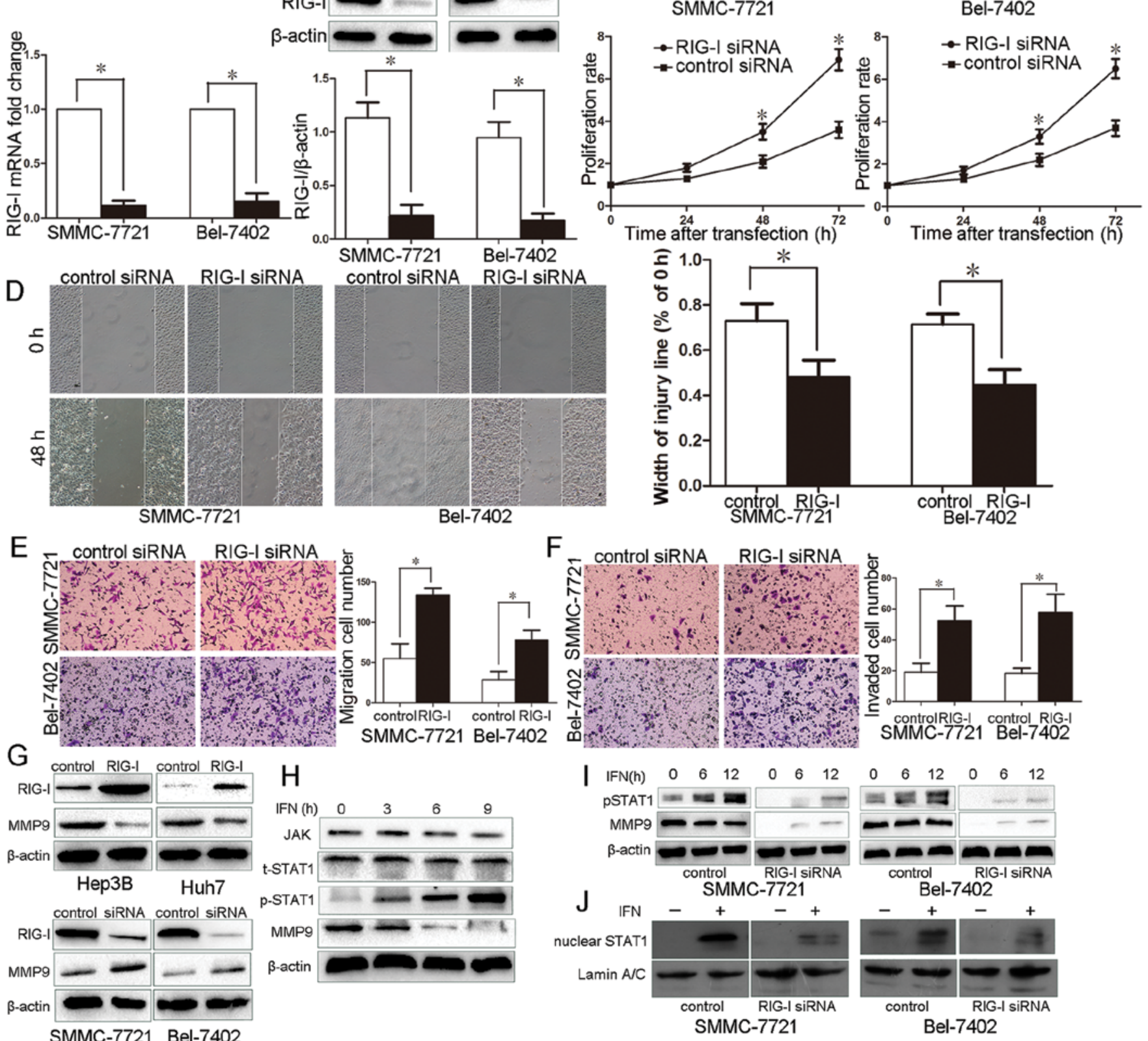

Bel-7402
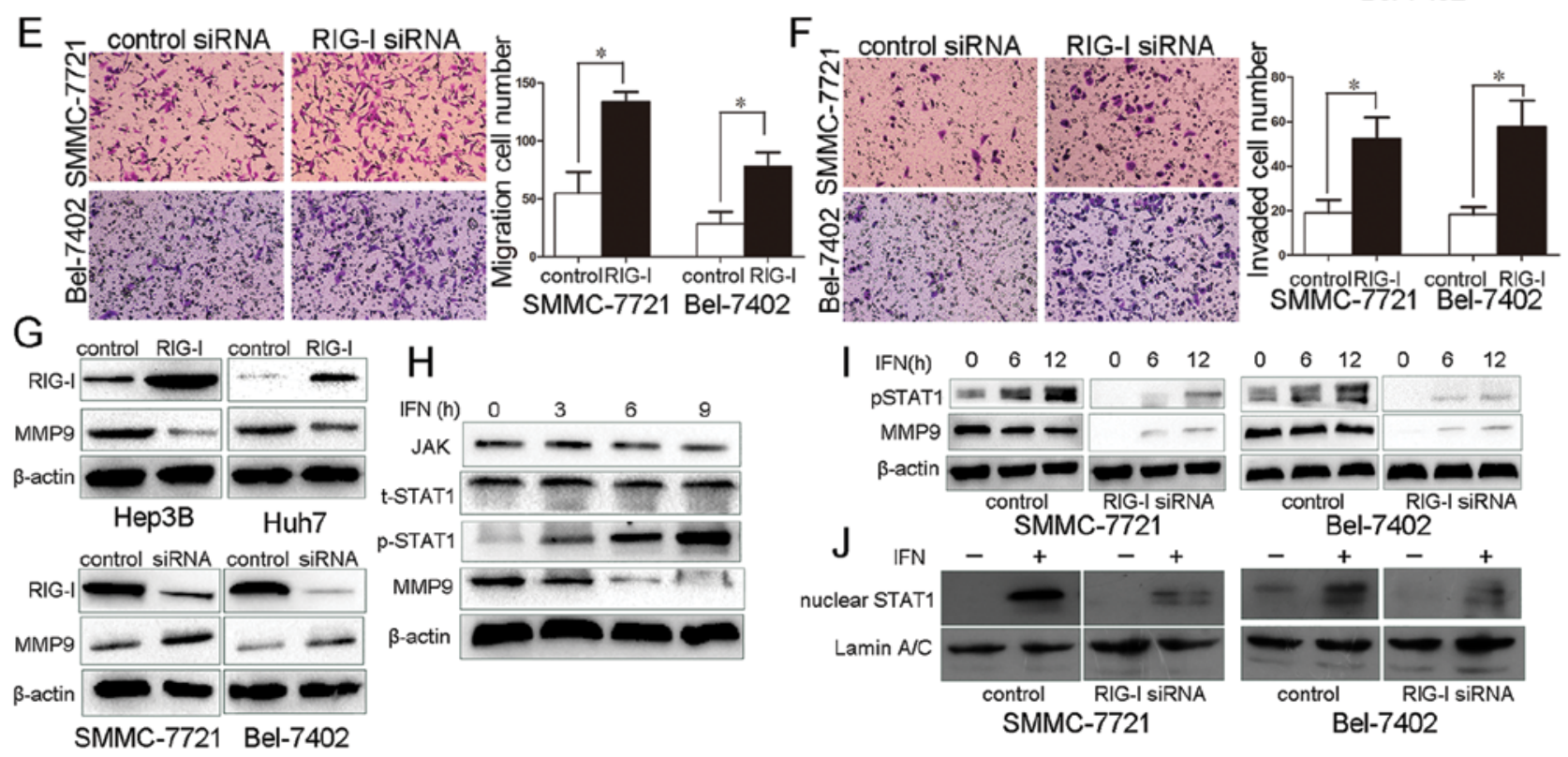

Figure 5. RIG-I downregulates MMP9 via STAT1 activation and strengthens the IFN-JAK-STAT effector signaling. (A) qRT-PCR confirms the knockdown in SMMC-7721 and Bel-7402 cells treated with RIG-I siRNA. (B) Western blotting confirmed RIG-I expression in SMMC-7721 and Bel-7402 cells. (C) Proliferation rates were assessed by MTT assay. The proliferation rate of SMMC-7721 and Bel-7402 cells treated with RIG-I siRNA was significantly greater than in control siRNA cells. (D) Wound healing assays showed that RIG-I knockdown enhanced the migration of SMMC-7721 and Bel-7402 cells. (E) RIG-I knockdown increased the number of migration. (F) RIG-I knockdown increased the number of invaded cells. (G) Expression of MMP9 protein was enhanced by RIG-I siRNA, overexpression of RIG-I significantly reduced MMP9 levels, as confirmed by western blot analysis. $\beta$-actin served as loading control. (H) IFN regulates MMP9 expression through the JAK-STAT pathway, and the inhibitory effect was time-dependent. (I) STAT1 phosphorylation determined by western blotting in SMMC-7721 and Bel-7402 cells transfected with RIG-I siRNA and stimulated with IFN- $\alpha$. (J) STAT1 nuclear translocation determined by western blotting in SMMC-7721 and Bel-7402 cells transfected with RIG-I siRNA and stimulated with IFN- $\alpha$. $\beta$-actin as internal control, and Lamin A/C as nuclear marker and internal control. ${ }^{*} \mathrm{P}<0.01$.

closure (Fig. 5D). Indeed, experiments of transwell chamber without Matrigel strengthen the results (Fig. 5E), tumor cells with RIG-I knockdown displayed a higher ability of migration. Consistently, the number of SMMC-7721 and Bel7402 cells that invaded through Matrigel was enhanced remarkably after RIG-I was downregulated (Fig. 5F). Collectively, these data demonstrated that RIG-I inhibited HCC cell proliferation, migration and invasion in vitro.
RIG-I regulates the expression of MMP9. Then we investigated a potential mechanism for RIG-I in HCC cell migration and invasion. Since MMP9 is a member of the matrix metalloproteinase (MMP) family, which has important roles in tumor cell migration and invasion (19-21), we assessed whether alteration of RIG-I expression in HCC cells influenced the protein expressions of MMP9. Results of western blotting confirmed that overexpression of RIG-I significantly reduced the expres- 


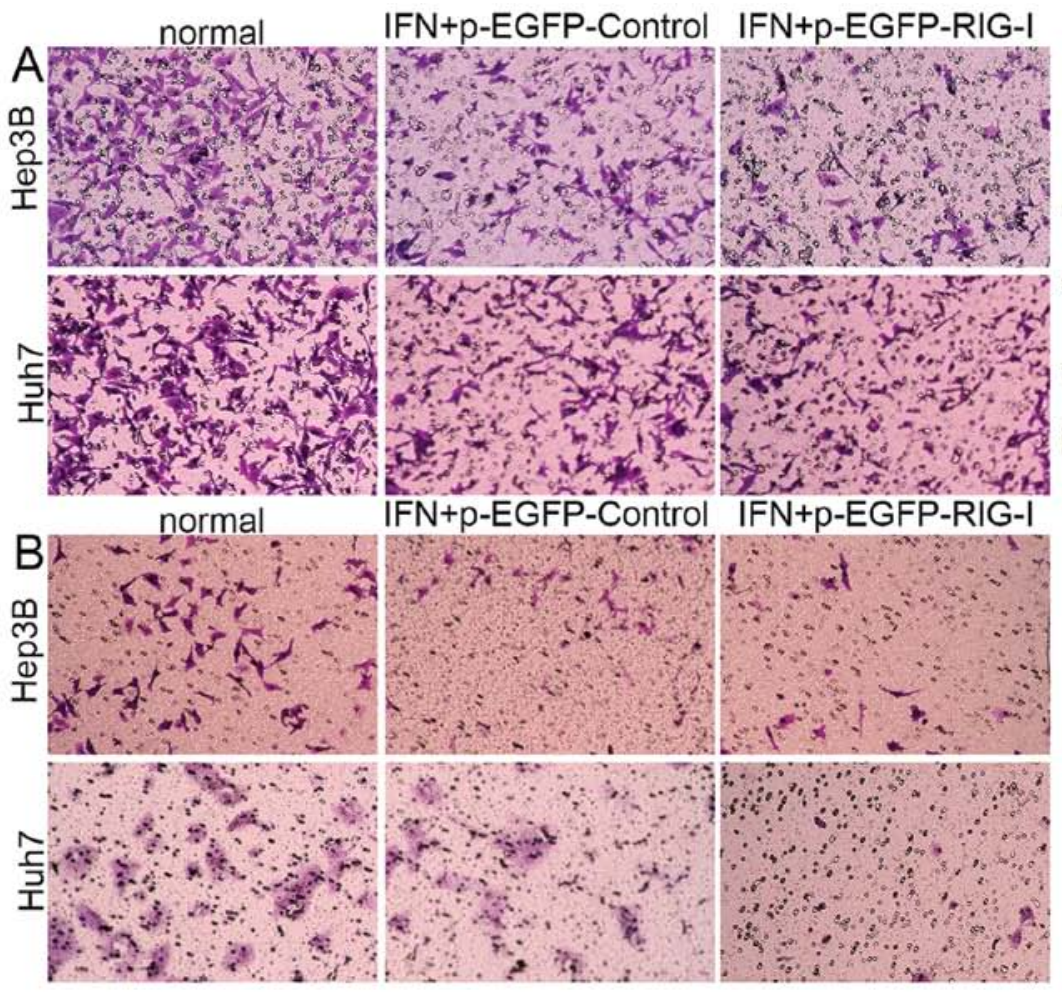

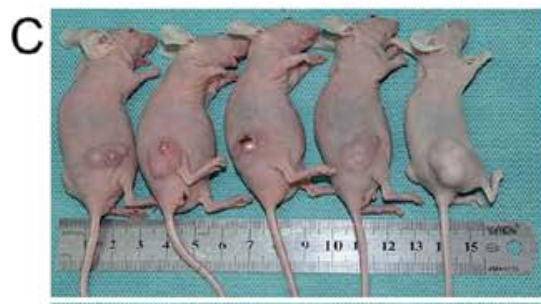
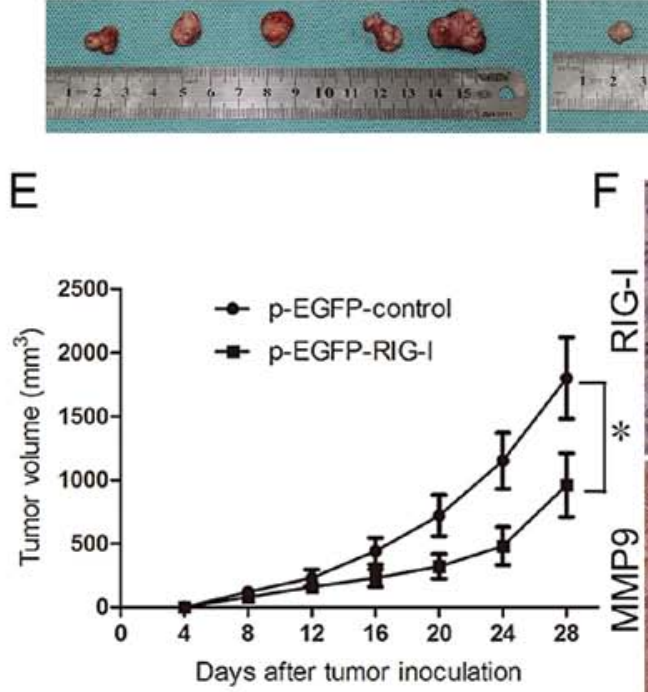

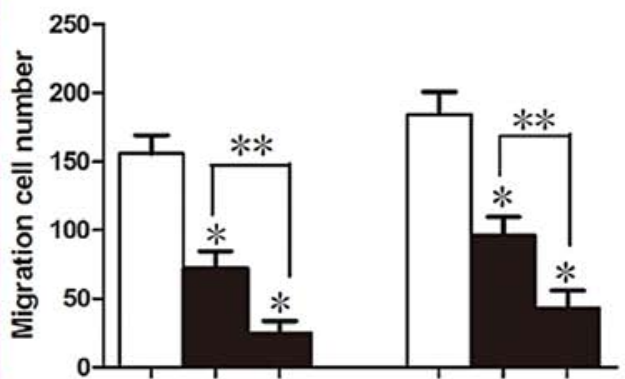

Hep3B

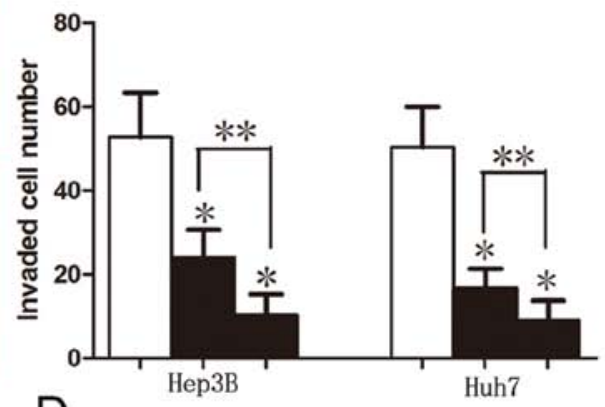

D

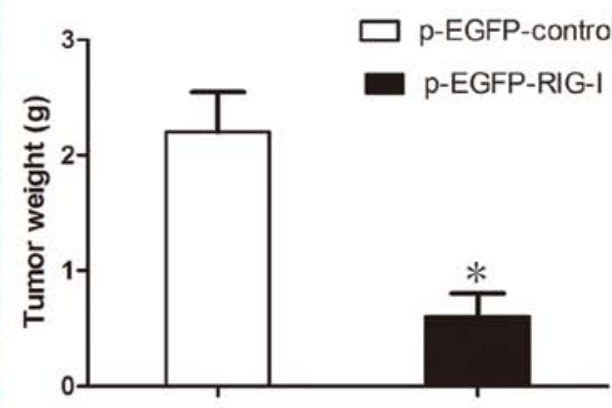

p-EGFP-RIG-I

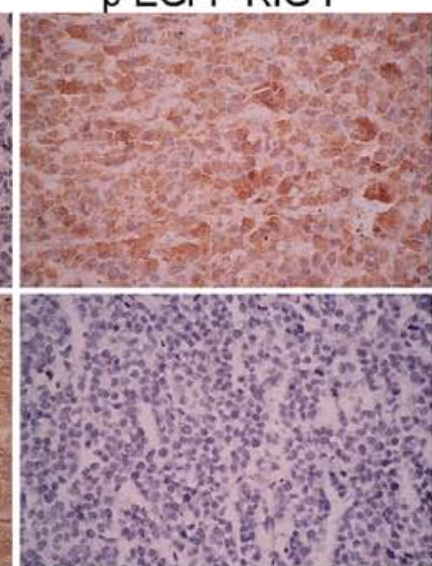

Figure 6. There was positive correlation between protein expression of RIG-I and MMP9 in Hep3B xenografts of nude mice. (A and B) RIG-I enhanced the effect of IFN- $\alpha$ inhibits the migration and invasion. (C and D) Exemplarily, tumor bearing mice and explanted tumors are depicted. Tumor size and weight was plotted in (C) and (D), respectively. (E) Control Hep3B cells (p-EGFP-control), RIG-I overexpressing Hep3B cells (p-EGFP-RIG-I), respectively, were implanted into nude mice via subcutaneous injection. Tumor nodules were measured using a caliper at different times after implantation. RIG-I overexpressing Hep3B cells exhibited a greater tumor-inhibiting effect compared with control cells. " $\mathrm{P}<0.01$ by two-way ANOVA. (F) Tumor nodules were subjected to immunohistochemical staining for RIG-I and MMP9. Representative immunostaining revealed that RIG-I overexpression significantly reduced the number of MMP9 positive cells. ${ }^{*} \mathrm{P}<0.01,{ }^{* *} \mathrm{P}<0.01$.

sions of MMP9. On the other hand, downregulation of RIG-I remarkably enhanced the MMP9 expression (Fig. 5G).
RIG-I downregulates MMP9 via STAT1 activation and strengthens IFN-JAK-STAT effector signaling. Interferon- $\alpha$ 
(IFN- $\alpha$ ), for the adjuvant therapy of HCC, Ugarte-Berzal et al verified that it could inhibit migratory and invasive capacity through downregulating MMP9 in chronic lymphocytic leukemia (22). Whether IFN- $\alpha$ could suppress MMP9 in HCC was still an unsolved question. As shown in Fig. 5H, we found the inhibitory effect of IFN- $\alpha$ on MMP9 was time-dependent in Hep3B. Since the IFN-JAK-STAT cascade is a classical pathway regulating MMP9 expression $(23,24)$, we investigated whether RIG-I modulated MMP9 expression through this pathway. Because RIG-I is one of the IFN-stimulated genes (18), which was significantly decreased in HCC, we investigated the association of RIG-I expression with the response to IFN- $\alpha$ therapy in HCC in vitro. The activation of the intracellular JAK-STAT pathway mediates the downstream expression upon IFN- $\alpha$ stimulation (7). In HCC cells, RIG-I knockdown inhibited IFN- $\alpha$-induced STAT1 activation and nuclear translocation (Fig. 5I and J), then contributed to downregulation of MMP9. These results suggested that RIG-I promoted the activation of IFN-JAK-STAT effector signaling in HCC cells. Additionally, we examined the effects of IFN on HCC cell migration and invasion (Fig. 6A and B). Consistently, RIG-I overexpression significantly decreased the migration and invasion compared to the single IFN effect. Collectively, these data demonstrate that RIG-I may enhance the antitumor effect of IFN- $\alpha$ by amplifying the IFN-JAK-STAT effector pathway in HCC cells by promoting STAT1 activation.

Positive correlation between protein expression of RIG-I and MMP9 in Hep3B xenografts of nude mice. To further prove our in vitro findings that RIG-I affected tumor growth by inhibiting MMP9, we investigated Hep3B cells in a subcutaneous tumor model. As shown in Fig. 6C and D, the size and weight of xenografts derived from Hep3B RIG-I cells (p-EGFP-RIGI group) was significantly smaller than that one from Hep3B control cells (p-EGFP-control group) at the 28th day after HCC cells injection. Consistently, tumor growth curves, generated over 28 days, revealed that RIG-I overexpression slowed down Hep3B tumor growth in mice (Fig. 6E). Additionally, we carried out IHC assay on xenograft tissues and found that RIG-I was expressed positively in all five xenografts from Hep3B RIG-I group and there was negative expression of MMP9 found in corresponding xenografts (Fig. 6F). The RIG-I protein in xenografts from Hep3B RIG-I group was detected in the cytoplasm, which was consistent with the finding of IHC assessment for HCC patients. The results of xenograft experiments confirmed that RIG-I suppresses the growth of hepatocellular carcinoma through MMP9.

\section{Discussion}

The dismal prognosis of HCC has been attributed to the post-surgical recurrence and metastasis-related de novo tumor development (25). Distinct HCC-associated proteins or microRNAs have also been proposed as biomarkers for patient prognosis $(26,27)$. However, scarce predictive biomarkers for HCC prognosis is available in clinic to date. Herein, we for the first time report the downregulation of RIG-I in HCC and clarified the correlation between RIG-I expression and the prognosis of patients. We initially investigated the expression of RIG-I protein in $80 \mathrm{HCC}$ patients using immunohisto- chemistry, qRT-PCR and western blotting. Our data showed that the expression of RIG-I was significantly lower in HCC compared with matched adjacent tissue. Furthermore, RIG-I expression was significantly correlated with tumor size, venous infiltration, Edmondson-Steiner grading and TNM tumor stage. Thus, this is the first report showing the clinical significance of RIG-I. Importantly, our data indicated that RIG-I positive expression was significantly correlated with a better 3-year survival for all HCC patients. Multivariate Cox repression analysis found that RIG-I was an independent factor in predicting both overall 3-year survival and disease-free survival in HCC patients. These results showed that the status of RIG-I was critical for prognosis determination in HCC patients. In this study, we confirmed that RIG-I suppressed tumor progression in HCC.

To investigate detailed mechanisms by which decreased expression of RIG-I contributed to the poor prognosis of patients with $\mathrm{HCC}$, we first evaluated the in vitro expression levels and cellular locations of RIG-I in 5 HCC cell lines and compared them with that of a normal liver cell line. Consistent with the low expression and cytoplasm location of RIG-I in HCC tissue, RIG-I was expressed in cytoplasm in these six HCC cell lines. As a tumor suppressor, we first demonstrated that RIG-I was a key tumor suppressor that regulated cell proliferation in different HCC cell lines, given that RIG-I mRNA expression correlated negatively with colony formation ability. Moreover, upregulation of RIG-I significantly suppressed HCC cell migration and invasion, whereas, when RIG-I was repressed by a RIG-I-specific siRNA, the tumor cell migration and invasion was markedly enhanced. Taken together, our results suggested that RIG-I played an important role in inhibiting HCC cell migration and invasion.

Mechanistically, it is well known that degradation of the extracellular matrix surrounding tumors is the most common feature of tumor cell invasion into surrounding tissues and early metastasis. Members of the MMP family secreted by invading tumor cells can degrade all essential components of the extracellular matrix. Thus, expression levels of these MMPs effectively reflect the aggressiveness of tumor cells and are associated with poor prognosis in various cancers. MMP9 is an important member of MMP family which is pivotal in degrading the basement membrane and has been proven to facilitate tumor invasion and metastasis in many types of cancer cells $(19,28,29)$. It has also been shown that the invasive and metastatic capabilities of HCC were restrained by suppression of MMP9 (30,31). Indeed, in this study, we found that inhibition of MMP9 expression was associated with the suppression of HCC cell migration and invasion in vitro after overexpression of RIG-I. In additional, we established an HCC xenograft mouse model and found that MMP9 was absent in xenografts with high RIG-I level. These results identify conclusively that MMP9 is downregulated by RIG-I in HCC. However, it is unknown how RIG-I regulates MMP9 expression. Further study will investigate this underlying mechanism.

Moreover, investigations of RIG-I in cancer also reported that RIG-I activation by its ligand pppRNA could induce apoptosis in melanoma cells and ovarian cancer cells (4). IFN had tumor-suppressive effects in cancer cells by IFN-JAK-STAT pathway to attenuate MMP9. The induced RIG-I expression and activation upon IFN- $\alpha$ stimulation could ensure a long- 
time IFN- $\alpha$ efficacy. We found that RIG-I was able to promote STAT1 activation and nuclear translocation, its interaction with STAT1 suggested that RIG-I might also function as an important intracellular regulator to modulate the MMP9 signaling pathway. Moreover, RIG-I antiviral signaling induced the production of type I IFN, which activated the downstream JAK-STAT pathway to induce IFN-stimulated genes expression (32). Here we found that RIG-I could amplify IFN- $\alpha$ effector signaling. It could be informative to study crosstalk between the RIG-I and IFN downstream pathways, especially the regulation of IFN downstream signaling by molecules in the RIG-I pathway.

The detailed mechanisms of RIG-I in preventing HCC carcinogenesis need to be further investigated. We postulate that host IFN- $\alpha$ may be important in the prevention of HCC carcinogenesis and the overexpression of RIG-I may enhance cellular response to IFN- $\alpha$, inhibiting HCC carcinogenesis. Moreover, as STAT1 activation by RIG-I may contribute to suppression of MMP9 in the HCC development. Future work will be needed to fully reveal the roles of RIG-I in the regulation of IFN- $\alpha$ effector signaling and antitumor responses.

\section{Acknowledgements}

This study was supported by grants from National Natural Scientific Foundation of China (nos. 81272645 and 81072052 to Qingguang Liu), Key Science and Technology Fund of Shaanxi Province to Tao Song (2010K01-131), and the Funds for Creative Research sponsored by The First Affiliated Hospital of Xi'an Jiaotong University to Zhikui Liu (14YB10).

\section{References}

1. El-Serag HB and Rudolph KL: Hepatocellular carcinoma: epidemiology and molecular carcinogenesis. Gastroenterology 132: 2557-2576, 2007.

2. Levine AJ and Puzio-Kuter AM: The control of the metabolic switch in cancers by oncogenes and tumor suppressor genes. Science 330: 1340-1344, 2010.

3. Kato H, Sato S, Yoneyama M, et al: Cell type-specific involvement of RIG-I in antiviral response. Immunity 23 19-28, 2005.

4. Besch R, Poeck H, Hohenauer T, et al: Proapoptotic signaling induced by RIG-I and MDA-5 results in type I interferonindependent apoptosis in human melanoma cells. J Clin Invest 119: 2399-2411, 2009.

5. Sun HC, Tang ZY, Wang L, et al: Postoperative interferon alpha treatment postponed recurrence and improved overall survival in patients after curative resection of HBV-related hepatocellular carcinoma: a randomized clinical trial. J Cancer Res Clin Oncol 132: 458-465, 2006

6. Clavien PA: Interferon: the magic bullet to prevent hepatocellular carcinoma recurrence after resection? Ann Surg 245: 843-845, 2007.

7. Taniguchi $\mathrm{T}$ and Takaoka A: A weak signal for strong responses: interferon-alpha/beta revisited. Nat Rev Mol Cell Biol 2: 378-386, 2001.

8. Der SD, Zhou A, Williams BR and Silverman RH: Identification of genes differentially regulated by interferon alpha, beta, or gamma using oligonucleotide arrays. Proc Natl Acad Sci USA 95: 15623-15628, 1998.

9. Schindler C, Shuai K, Prezioso VR and Darnell JE Jr: Interferondependent tyrosine phosphorylation of a latent cytoplasmic transcription factor. Science 257: 809-813, 1992.
10. Heinrich PC, Behrmann I, Haan S, Hermanns HM, MullerNewen $G$ and Schaper F: Principles of interleukin (IL)-6-type cytokine signalling and its regulation. Biochem J 374: 1-20, 2003.

11. Chawla-Sarkar M, Lindner DJ, Liu YF, et al: Apoptosis and interferons: role of interferon-stimulated genes as mediators of apoptosis. Apoptosis : an international journal on programmed cell death 8: 237-249, 2003.

12. Bromberg J: Stat proteins and oncogenesis. J Clin Invest 109: 1139-1142, 2002.

13. Yu H, Kortylewski M and Pardoll D: Crosstalk between cancer and immune cells: role of STAT3 in the tumour microenvironment. Nat Rev Immunol 7: 41-51, 2007.

14. Jonasch $\mathrm{E}$ and Haluska FG: Interferon in oncological practice: review of interferon biology, clinical applications, and toxicities. Oncologist 6: 34-55, 2001.

15. Bekisz J, Baron S, Balinsky C, Morrow A and Zoon KC: Antiproliferative properties of type I and type II interferon. Pharmaceuticals 3: 994-1015, 2010.

16. Rao JS, Yamamoto M, Mohaman S, et al: Expression and localization of $92 \mathrm{kDa}$ type IV collagenase/gelatinase B (MMP-9) in human gliomas. Clin Exp Metastasis 14: 12-18, 1996.

17. Liotta LA, Tryggvason K, Garbisa S, Hart I, Foltz CM and Shafie S: Metastatic potential correlates with enzymatic degradation of basement membrane collagen. Nature 284: 67-68, 1980.

18. Hou J, Zhou Y, Zheng Y, et al: Hepatic RIG-I predicts survival and interferon-alpha therapeutic response in hepatocellular carcinoma. Cancer Cell 25: 49-63, 2014.

19. Egeblad M and Werb Z: New functions for the matrix metalloproteinases in cancer progression. Nat Rev Cancer 2: 161-174, 2002.

20. Bjorklund M and Koivunen E: Gelatinase-mediated migration and invasion of cancer cells. Biochim Biophys Acta 1755: 37-69, 2005.

21. Lamar JM, Pumiglia KM and DiPersio CM: An immortalizationdependent switch in integrin function up-regulates MMP-9 to enhance tumor cell invasion. Cancer Res 68: 7371-7379, 2008.

22. Ugarte-Berzal E, Redondo-Munoz J, Eroles P, et al: VEGF/ VEGFR2 interaction down-regulates matrix metalloproteinase-9 via STAT1 activation and inhibits $B$ chronic lymphocytic leukemia cell migration. Blood 115: 846-849, 2010.

23. Ma Z, Qin H and Benveniste EN: Transcriptional suppression of matrix metalloproteinase- 9 gene expression by IFN-gamma and IFN-beta: critical role of STAT-1alpha. J Immunol 167: 5150-5159, 2001

24. Yen JH, Kong W and Ganea D: IFN-beta inhibits dendritic cell migration through STAT-1-mediated transcriptional suppression of CCR7 and matrix metalloproteinase 9. J Immunol 184: 3478-3486, 2010.

25. Bruix J, Sherman M and American Association for the Study of Liver Disease: Management of hepatocellular carcinoma: an update. Hepatology 53: 1020-1022, 2011.

26. Lee JS, Chu IS, Heo J, et al: Classification and prediction of survival in hepatocellular carcinoma by gene expression profiling. Hepatology 40: 667-676, 2004.

27. Budhu A, Jia HL, Forgues M, et al: Identification of metastasisrelated microRNAs in hepatocellular carcinoma. Hepatology 47: 897-907, 2008.

28. Deryugina EI and Quigley JP: Matrix metalloproteinases and tumor metastasis. Cancer Metastasis Rev 25: 9-34, 2006.

29. Fingleton B: Matrix metalloproteinases: roles in cancer and metastasis. Front Biosci 11: 479-491, 2006.

30. Kessenbrock K, Plaks V and Werb Z: Matrix metalloproteinases: regulators of the tumor microenvironment. Cell 141: 52-67, 2010.

31. Yang P, Yuan W, He J, et al: Overexpression of EphA2, MMP-9, and MVD-CD34 in hepatocellular carcinoma: implications for tumor progression and prognosis. Hepatol Res 39: 1169-1177, 2009.

32. Takeuchi $\mathrm{O}$ and Akira $\mathrm{S}$ : Pattern recognition receptors and inflammation. Cell 140: 805-820, 2010. 